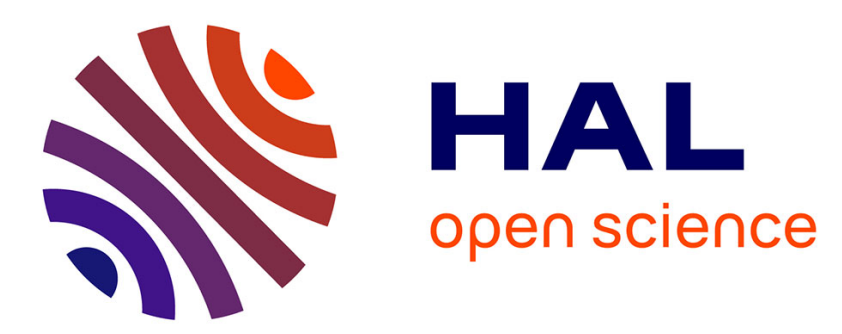

\title{
A possibilistic-valued multi-criteria decision-making support for marketing activities in e-commerce: Feedback Based Diagnosis System
}

\author{
Afef Denguir, Jacky Montmain, Gilles Mauris
}

\section{- To cite this version:}

Afef Denguir, Jacky Montmain, Gilles Mauris. A possibilistic-valued multi-criteria decision-making support for marketing activities in e-commerce: Feedback Based Diagnosis System. European Journal of Operational Research, 2009, 195 (3), pp.876-888. hal-00353774

HAL Id: hal-00353774

https://hal.science/hal-00353774

Submitted on 27 May 2021

HAL is a multi-disciplinary open access archive for the deposit and dissemination of scientific research documents, whether they are published or not. The documents may come from teaching and research institutions in France or abroad, or from public or private research centers.
L'archive ouverte pluridisciplinaire HAL, est destinée au dépôt et à la diffusion de documents scientifiques de niveau recherche, publiés ou non, émanant des établissements d'enseignement et de recherche français ou étrangers, des laboratoires publics ou privés. 


\title{
A possibilistic-valued multi-criteria decision-making support for marketing activities in e-commerce: Feedback Based Diagnosis System
}

\author{
Afef Denguir-Rekik ${ }^{\mathrm{a}, \mathrm{b}}$, Jacky Montmain ${ }^{\mathrm{b}, *}$, Gilles Mauris ${ }^{\mathrm{a}}$ \\ ${ }^{\text {a }}$ LISTIC - Université de Savoie, BP 806 74016, Annecy, France \\ ${ }^{\mathrm{b}}$ LGI2P - Ecole des Mines d'Alès, Site EERIE-Parc Scientifique G. Besse, 30035 Nîmes Cedex, France
}

\begin{abstract}
In this paper we propose a multi-criteria decision making support system, called a "Feedback Based Diagnosis System" (FBDS), to aid the marketing team of an e-commerce (EC) organisation in its activities. The FBDS database is composed of customers' satisfaction measures. These measures are related to the different services an EC offers to its customers. Thus, they constitute a multi-criteria (MC) evaluation of EC performances. In the general framework of recommender systems, these available MC evaluations are considered as useful information for other customers to help them to objectively, rationally and exhaustively assess and compare the numerous ECs among the ones likely to meet their needs. Our FBDS is not concerned with improving or automating such a recommendation process for customers. Indeed, it is merely EC management team oriented. In fact, the MC feedback database is used to diagnose the EC health and improve its strategy. In the proposed FBDS, a possibilistic framework is combined with the multi criteria representation to capture the variability and the divergence of customers' evaluations w.r.t. each criterion. Then, an aggregation based on a weighted arithmetic mean (WAM) is proposed to obtain a synthetic appraisal of ECs. The WAM aggregation models the strategy agreed on by the EC management team. Computing the synthesis score of an EC consists in propagating the uncertainty related to its partial scores through the WAM. The possibilistic representation guarantees that no information is lost in the collective evaluation process by the consumers' community. However, diagnosis indicators are finally proposed to the marketing team to make the interpretation of some possibilistic results more comprehensive when necessary.
\end{abstract}

Keywords: Decision making support system; Possibility theory; Knowledge-based recommender systems; Diagnosis indicators; E-commerce; Customers' satisfaction measures

\section{Introduction and outline of our research}

The last decade has seen an explosion in the growth and the use of the Internet. New terms have appeared to more accurately distinguish the different types of business transactions that take place on the Internet. One of these new terms is "e-commerce" (EC). The EC can be defined as the exchange transactions which take place over the Inter-

\footnotetext{
* Corresponding author. Tel.: +3346638 70 58; fax: +33 466387074 .

E-mail addresses: afef.denguir@univ-savoie.fr (A. Denguir-Rekik), jacky.montmain@ema.fr (J. Montmain), mauris@univ-savoie.fr (G. Mauris).
}

net primarily using digital technology. This encompasses all activities supporting market transactions including marketing, customers' support, delivery and payment (Schniederjans and Cao, 2002). One important problem in EC marketing activities is the process of building and maintaining customer relationships through online activities to facilitate the exchange of ideas, products, and services that satisfy the goals of both parties (Kwan et al., 2005). Thus EC marketing teams devote most of their time to develop indicators to efficiently monitor their activities and adapt their business strategy dynamically.

The specificity of e-marketing is related to the Internet and the WWW technologies. Indeed, faced with important 
amounts of information and a multitude of potential choices on the Web, indecisive Internet consumers tend to turn to the opinions and experiences of other customers to make their choices. A new type of websites has thus appeared to support this emerging sharing process. They propose to mediate, support, or automate the everyday process of sharing recommendations between cyber-consumers' communities (Mcnee et al., 2003; Schafer et al., 2001).

The so-called recommender systems (RS) are based on various computational techniques and data about consumers' feedbacks, preferences or behaviours as will be discussed in Section 2. However, in all cases: (1) RSs have at their disposal a huge amount of information about consumers' practices, preferences, etc.; (2) RSs are consumer-oriented: recommendation consists in proposing the most suitable item to meet a specific consumer's need using information about other consumers. In this paper, RS databases are diverted from their initial aim, and thus the proposed Feedback Based Diagnosis System (FBDS) is dedicated to the marketing team. It is explained how the marketing team of an EC can derive benefits from the available RS feedback databases to diagnose inefficient or weak dimensions of its activities and then adapt its business strategy.

We pay a particular attention to RS databases where customers' feedbacks are accompanied with evaluations of tested e-retailers. Furthermore, these evaluations are often organized relatively to a predefined subset of criteria such as Range of Services/Products, Purchase Tracking Function and Security of Payment, etc. Then, the collection of evaluations gathered on a RS is useful as a working base to the marketing team of an EC to on line diagnose bad or decreasing results w.r.t. specific criteria of its activity. From this viewpoint, the set of evaluations gathered by the RSs provides any EC with a performance measurement system of its activity. The design and use of performance measurement systems (PMSs) have received considerable attention in recent years. Indeed, industrial performances are now defined in terms of numerous criteria to be synthesized for overall improvement purposes. The analysis of the literature leads to the conclusion that most of the proposed approaches deal with a qualitative approach of this multi-criteria issue. But only a few quantitative models for PMSs have been proposed in order to better monitor the continuous improvement cycle (Berrah et al., 2004, 2008). Designing such a quantitative PMS often appears as a thorny task in many industrial sectors because few performance measurements are available, their appraisals are only qualitative and/or subjective and their real-time updating appears as a demanding task. In the field of EC companies, it can be considered that the PMS is "offered" by RSs that propose evaluative feedbacks. The richness of such RS databases provides a natural PMS to any EC. The problems of the subjective evaluation of an indicator and of its updating are inhibited by the number of potential evaluators of the WWW. This digression w.r.t. industrial PMSs puts our work back in an industrial issue that can largely reach beyond the marketing activities in e-com- merce. However, we believe that the specificity of performance management in e-commerce is related to the nature, the number and updating frequency of the performance indicators by the e-consumers' community. This particularity deserves careful thoughts that will be discussed in this paper.

Thus, we propose a range of solutions to facilitate the marketing decision-making in e-commerce in an uncertain environment. First, our study focuses on the use of customers' satisfaction measures available on the Web to build comprehensive decision-making indicators for marketing strategies. Secondly, our contribution concerns the definition of a possibilistic frame to capture the dispersion and the imperfection of the evaluations related to the feedbacks of the customers. Finally, we propose the conception and the development of a software tool to support marketing activities. In particular, choice justification and traceability functions are emphasized.

The paper is organized as follows. Section 2 explains how customer satisfaction collected on line generally for customer recommendation purposes can be used to aid a marketing team in its diagnosis and decisions. The case of ciao.com is exposed. It then constitutes a running example showing in a step by step fashion the application of our method. Section 3 defines the possibilistic tools used to represent the users' opinions. In particular, quantitative easily interpretable indicators are proposed to describe a possibility distribution, for both mono modal and bimodal cases. The indicators discussed in this paper are more particularly dedicated to the detection and the diagnosis of imprecision and divergence aspects in customers' appraisals. Section 4 presents the multi-criteria aggregation through a weighted arithmetic mean (WAM) in uncertain environment. The propagation of the indicators through the WAM is detailed and their use to support decision makers is presented. Finally a discussion and a conclusion are drawn.

\section{Feedback based recommendation systems and diagnosis tools for EC managers}

In this section, we first introduce the notion of customer satisfaction measure. It will constitute the basic concept that supports our evaluation system. Then, our approach is resituated in the general framework of recommender systems on the Net. Its specificity is related to its aim: unlike to most of the RSs, it is not customer-oriented but dedicated to a marketing team which tries to analyse the performances of its EC for diagnosis purposes. It has major consequences on the multi-criteria analysis of EC activities. The last subsection gives our viewpoint. The explanation and diagnosis functionalities of our software tool are emphasized.

\subsection{The customer satisfaction measure}

The service quality is a major concern in management as well as in marketing. Moreover, the particularly intense 
competition over the web imposes permanent efforts of adaptation and optimization of the website, the offers and services. E-commerce marketing is submitted to more drastic temporal constraints and limited response times to attend to customers' requirements. Indeed, the loyalty of customers and the profitability of an e-commerce company are maximized when meeting or exceeding customers' requirements. Thus, customer satisfaction measurement enables EC managers to: (i) accurately identify customers' requirements and their relative importance; (ii) understand how customers perceive the EC and whether its performance meets their requirements; (iii) pinpoint the priorities for improvement; (iv) define objectives of service improvement and follow the progress towards a customer satisfaction index; (v) increase profits through improved customer loyalty. Besides, to establish the customer viewpoint, it is necessary to identify the factors which predominate in their evaluations of the EC.

Therefore, different approaches dealing with the assessment of customer satisfactions already exist (Grigoroudis and Siskos, 2002). Let us also mention data analysis techniques (cluster analysis, probability plotting method), consumers behavioural analysis, MUlticriteria Satisfaction Analysis (which is a preference disaggregation model following an ordinal regression analysis), etc. Other works dealing with the management of qualitative customers' satisfaction measures are presented in Zollo et al. (1999). Zollo et al. (1999) assist the customers in expressing and explaining their evaluation during the more or less "indepth" interviews (Leadershipfactor, 2004). But as these approaches are time consuming, only a panel of consumers is considered.

Thus, our approach is based on feedback evaluations given by the customers on line after a purchase or a request. The current models generally do not consider the imprecision in customers' evaluations: the variability or the controversy phenomena of customers' degrees of satisfaction are not highlighted. Furthermore, these approaches rather concern the identification of a company strategy from the modelling of customers' satisfaction, whereas we are concerned with how to improve the results of an EC company in the framework of an already identified and acknowledged strategy.

\subsection{Main existing recommendation systems ( $R S)$}

Nowadays, due to the Internet and the EC progress, a lot of information on customers' satisfaction feedbacks is now available on-line. The EC marketing teams have a direct access to this data; however, they are faced with an overload of data which is neither formatted, nor formalized. Rough data are to be sorted out, managed and analysed. These problems gave birth to recommender systems (Schafer et al., 1999; Schafer, 2005; Terveen and Hill, 1991).

The large amount of digital information makes it more and more difficult for a person to collect, filter, evaluate and use information available on the Internet for problem solving (Zhaohao and Gavin, 2004). In addition, the wide range of Ecs' prevents the customers from objectively, rationally and exhaustively assessing and comparing the panel of web sites all likely to meet their needs (Mcnee et al., 2003; Terveen and Hill, 1991). That is why nowadays customers are first turning to recommender systems (RS) before performing an e-purchase. The principle of e-recommendation is to provide the customer, confronted with this dilemma, with opinions or feedbacks of other customers who have already tested and evaluated the EC sites he does not manage to differentiate by himself.

Several algorithmic approaches have already been applied for implementing precise and effective RSs (Schafer, 2005). The first RSs were the "content based filtering systems" designed to reduce the overload of the information in textual domains by the use of matching functions comparing formal representations of texts (Lang, 1995; Pazzani et al., 1996). The "collaborative filtering" (CF) RSs attempt to improve the filtering for a particular user by considering the information provided by other users. The "memory-based collaborative filtering" operates over the entire user data base to make the recommendation by using a correspondence with the profile of $A$ and all the other evaluators' profiles (Mukherjee et al., 2001; Herlocker et al., 2000). In contrast, the "model-based collaborative filtering" uses only the user data base to learn a model which is then used to make the recommendation (Breese et al., 1998). In terms of customers' satisfaction, it is important to notice that both these systems of CF supply direct measures of satisfaction. The profile of a costumer is based upon the way he has evaluated a set of items.

Many RSs use Data Mining techniques. In such RSs, recommendations rely, for example, on the knowledge the RS has acquired from actions and characteristics of customers. These systems are often based upon the development of users' profiles (Schafer, 2005). It is still an indirect measure of customer satisfaction.

The techniques developed for RSs are various; the notion of neighbouring may get several meanings. The only common point to all these systems of recommendation is their finality: they support a customer choosing a product or a supplier of products. Some of them use direct measures of customers' satisfaction; others rely on indirect measures of satisfaction. As for "collaborative Filtering" RSs, our approach needs direct measures of satisfaction. However, it neither relies on data mining techniques nor on similarity calculus. Furthermore, even when direct measures of satisfaction are available in RSs, appraisals are rarely related to a multi-criteria analysis. For this reason, we consider in this paper a particular website proposing e-recommendation from direct measures of customers' satisfaction according to a predefined set of criteria. This website is "ciao.com" and it is one of Europe's leading consumer-oriented shopping comparison portals (http:// www.ciao.co.uk/shopping partners). Each customer member of "ciao.com" - is usually invited to attribute 
partial scores to an e-commerce website $\left(s^{l}\right)$ according to a set of criteria such as range of services/products, product and service pricing, purchase tracking function, security and privacy statement, etc., after his purchasing.

\subsection{From a recommendation system to a diagnosis support system}

"ciao.com" is clearly dedicated to customers; we thus suggest reorienting and extending its functionalities to support marketing activities. It is based upon a feedbacks database. Direct measures of satisfaction are exploited in a multidimensional evaluation space. Each measure of satisfaction is related to one of the criteria of the evaluation process. Thus, in this paper, we consider a new kind of RS, called "Feedback Based Diagnosis System" (FBDS). Unlike the existing RS mentioned above, a FBDS is not dedicated to customers but to EC managers (Fig. 1).

Thus, our aim is not providing customers with the most appropriate EC according to their preferences or their habits, but diagnosing and explaining critical results of the EC to the marketing team. As a consequence, the only reason our FBDS is related to RSs is that the base of costumers is the knowledge base of both systems. cia.com and the FBDS both use the same customers' feedbacks databases. In cia.com, direct measures of customers' satisfaction are available since customers are invited to provide evaluations for all the sites they used. Furthermore, these evaluations refer to multi-criteria assessments. The criteria are imposed by cia.com a priori. In cia.com, an evaluated $\mathrm{EC}_{l}$ is thus characterized by its partial score vector $\left(m_{1}^{l}, \ldots, m_{n}^{l}\right)$ where $m_{i}^{l}$ is the arithmetic mean upon all the available evaluations in the database concerning $\mathrm{EC}_{l}$ w.r.t. criterion $i$. Let us still notice that criterion $n$ is named "overall impression". $m_{n}^{l}$ is supposed to be a synthesis score. Nevertheless, the notion of aggregation is not referred to in cia.com. Indeed, in cia.com, which is customer-oriented, the way the overall impression score is filled is particular to each customer. It means that in the framework of customer-oriented RSs, aggregation is meaningless because each customer has his/her own aggregation operator (his/her own priorities, relative importance, etc.). On the contrary, the FBDS is addressed to the marketing team of an EC. Contrary to the set of individual customers, the team is supposed to share a unique objective for the EC. The members of the marketing team implicitly share a common strategy for their EC; they perfectly know which criteria of its activities are more or less fundamental, critical, etc. In other words, they would be able to affect a relative importance to each criterion of the evaluation space. Attributing an overall score to an EC in the FBDS framework is then meaningful because the aggregation step models the evaluation strategy of the marketing staff. For the sake of simplicity, only WAM aggregations are considered in this paper (more sophisticated operators have been studied in Denguir et al. (2006a). The WAM aggregation models the marketing team evaluation strategy relatively to the set of criteria. The WAM aggregation that is proposed in the FBDS must not be confused with the arithmetic mean on the criterion by criterion customers' evaluations that are proposed in ciao.com.

Furthermore, no aspect concerning the uncertainty inherent to the various opinions is taken into account in classical RSs. In particular, uncertainty is related to the dispersion and the imprecision of the scores given by the customers' community to a given EC: everyone has his/her own experience and assesses the EC according to his/her own scale of values. The mere statistical average of evaluations currently proposed by ciao.com thus constitutes a drastic information reduction. The assumption behind this synthetic "average indicator" is that it is actually representative of the whole community only when it results from a weak dispersion around this value (there is a genuine consensus around it). However, the average rate can either result from a wide dispersion of scores which signifies that the opinions are not clear-cut and the evaluation is rather imprecise (all the opinions have been reported), or from conflicting viewpoints that should be represented by a multimodal distribution and for which the average value is not necessarily a relevant indicator. Conventional aggregation models from $\mathbb{R}^{n} \rightarrow \mathbb{R}$ can thus be misleading because they do not give any idea about the variability or the controversial aspects of the customers' opinions.

In this view, we propose to adopt a possibilistic framework (see the details in Section 3): the evaluation of an EC according to a criterion is no longer a single precise score but a distribution representing the whole set of customers' opinions w.r.t. this criterion. Then, the global evaluation of an EC is seen as a possibility distribution that results from the propagation of the partial distributions related to each evaluation criterion. From this viewpoint, all information contained in the feedback knowledge base

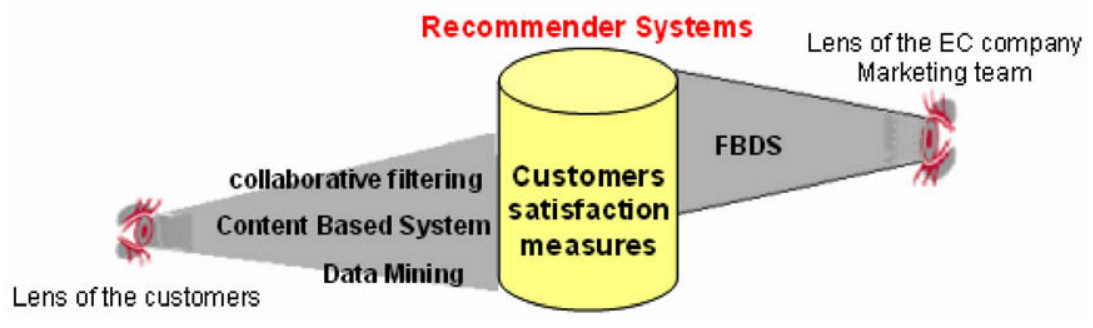

Fig. 1. Different lenses of RS. 


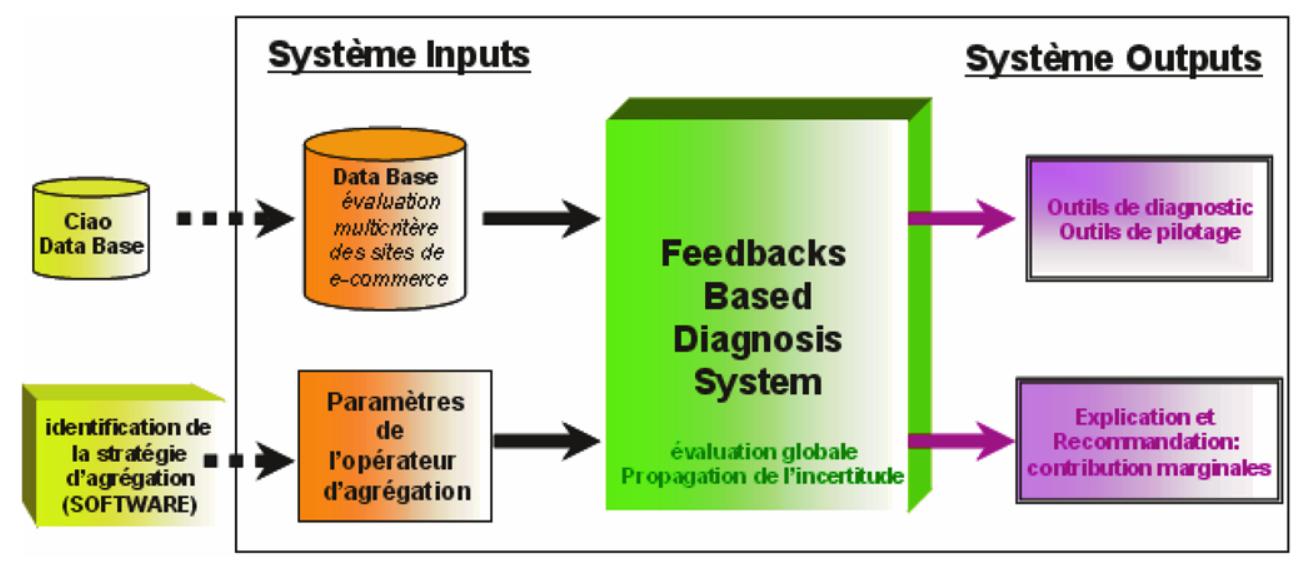

Fig. 2. The FBDS functionalities.

is explicitly taken into account in the EC evaluation: there is no loss of information concerning the variability and the controversy aspects of the appraisal in such an evaluation process.

\subsection{A FBDS based on ciao.com}

The e-recommender website ciao.com requires some adaptations before being used for diagnosis purposes by a marketing team. From this viewpoint, ciao.com suffers from the following shortcomings: (i) no aggregation model reflecting the EC managers' strategy is proposed; an EC is characterized by a partial scores vector $\left(m_{1}^{k}, \ldots, m_{n}^{k}\right)$, but all the criteria of the evaluation are of the same importance, and do not reflect the activities that should have priority for the management staff; (ii) variability is not considered in the evaluation process; nothing is proposed in the recommendation about the dispersion, the imprecision or the divergence of the evaluations attributed by the customers although this kind of information can represent a great added value in terms of decision-making.

The ciao.com evaluation database is already partitioned relatively to the criteria predefined by ciao. The first thing to do concerning the already structured evaluations database is to formalize the way we process with "stars" (labels used by customers to attribute their partial scores) when a numerical aggregation has to be performed. The second step is to model the strategy of the management team through an aggregation operator (a WAM in this paper); here, the aggregation model reflects the priorities, preferences or selection strategy, it is thus an indispensable step in the FBDS frame, whereas in conventional RSs, the aggregation step is meaningless because each customer has his/her own choice strategy, ones own centres of interest. Finally, the aggregation must be extended to the case when the WAM inputs are imprecise and uncertain scores (possibility distributions). The set of customers' evaluations w.r.t. a criterion $i$ is modelled by a possibility distribution and the aggregation process must thus deal with partial distributions. Aggregating the partial distributions consists in propagating uncertainty in evaluations from cri- terion distributions to the overall distribution resulting from the WAM operator.

Based on the evaluation database provided by ciao.com, a prototype of the FBDS has been developed. It proposes a multi-criteria evaluation and an aggregation step, ranking and comparison of ECs, explanation and justification functionalities of the established EC ranking. The evaluative feedbacks database of ciao.com can be partitioned according to the set of criteria. It obviously constitutes a mine of information for the marketing activities of an EC. Indeed, these experience feedbacks related to all the evaluation criteria of the ECs activities represent a useful working base to better meet customers demands and remain competitive in real-time, to identify weaknesses and strengths of ECs. The analysis of the ECs own evaluation or that of its competitors by customers provides relevant indicators to monitor and diagnose the results of the EC (Fig. 2).

The FBDS tries to give some elements of response to the issues mentioned above. A formal frame is proposed to model the relationship between a site of e-recommendation (ciao.com) and its EC partners. Each one of these two actors has his own interests and needs in this relation:

-ciao.com constitutes a network of experts in e-purchasing to mediate the process of sharing recommendations. It aims to be the most comprehensive source of shopping intelligence on the web to keep users and EC partner's loyalty by providing economic intelligence, surveys and experience feedback.

-The EC partners' goal is to enhance their performances: they thus use the evaluations, criticisms and rankings provided by ciao.com as performance indicators to improve their business capacities.

\section{Possibilistic framework and decision aiding indicators}

\subsection{Notations}

In the remaining of the paper, the following notations are considered (see Table 1): 
Table 1

Possibilistic notations

\begin{tabular}{ll}
\hline$s^{l}$ & the evaluated e-commerce $(l=1,2, \ldots, q)$ \\
$m^{l}$ & global evaluation of $s^{l}$ (customer's overall degree of satisfaction) \\
$m_{i}^{l}$ & partial evaluation of $s^{l}$ according to criterion $i(i=1,2, \ldots, n)$ \\
{$\left[m_{1}^{l}, m_{2}^{l}, \ldots, m_{n}^{l}\right]$} & $\begin{array}{l}\text { evaluation profile associated to } s^{l} \\
\text { partial evaluation distribution of an e-commerce website according to criterion } i(i=1,2, \ldots, n) \text { (mono modal or bimodal distribution) }\end{array}$ \\
$\pi_{i}$ & aggregated (overall) evaluation distribution of an e-commerce website (mono modal or multi-modal distribution) \\
$\pi_{\mathrm{ag}}$ & the possibility measure of the set $A$ for a possibility distribution $\pi: \Pi(A)=\sup _{x \in A} \pi(x)$ \\
$\Pi(A)$ & the necessity measure of the set $A$ for a possibility distribution $\pi: N(A)=1-\sup _{x \in \bar{A}} \pi(x)$ \\
$N(A)$ & $P(A)$ the probability measure of the set $A$ for a probability distribution $p: P(A)=\int_{x \in A} p(x) \mathrm{d} x$ \\
$P(A)$ & the cumulative probability function associated to a probability distribution $p: F(x)=\int_{-\infty}^{x} p(x) \mathrm{d} x$ \\
$F$ & the lower probability measure of the set $A$ defined by the necessity measure $N(A)$ \\
$P^{*}(A)$ & the lower probability measure of the set $A$ defined by the necessity measure $N(A)$ \\
$P^{*}(A)$ & mean value of the upper probability distribution belonging to the family defined by a possibility distribution \\
$E^{*}(\pi)$ & $\pi: E^{*}(\pi)=\int_{-\infty}^{+\infty} x \mathrm{~d} F_{*}(x) \mathrm{d} x$ \\
$E^{*}(\pi)$ & mean value of the lower probability distribution belonging to the family defined by a possibility distribution $\pi: E_{*}(\pi)=\int_{-\infty}^{+\infty} x \mathrm{~d} F^{*}(x) \mathrm{d} x$
\end{tabular}

\subsection{Possibilistic framework}

As explained in the introduction, a major limitation of classical RSs' evaluations lies in the fact that the arithmetic mean of the evaluations of all the RS members are drastic reductions to average assessments and they prevent decision-makers from really appreciating the uncertainty inherent to any collective evaluation: they cannot have any idea about the variability and controversial aspects of the evaluations.

With that in mind, we propose that the evaluations relative to any evaluation criterion of an EC are no longer deceptively considered as precise real values - the arithmetic mean upon the customers' ratings - but as possibility distributions. This possibility representation will not be deeply discussed here versus a probability one. We can merely claim that the possibility theory is useful in any problem where heterogeneous uncertain and imprecise data are concerned, e.g. subjective, linguistic-like evaluations and statistical data, which is the case in our application context. Moreover, for many criteria, only a small number of experience feedbacks are available; as a consequence, the identification of a specific probability distribution (Gaussian, triangular, uniform, etc.) is not always straightforward and several probability distributions may be suitable for the same series of scores.

The set of scores relative to a given criterion for a given EC can directly be collected under the form of a histogram. The histogram must then be transformed into a possibility distribution. The latter can be built using either a probability-possibility transformation (Dubois et al., 2004) or directly from statistical overlapped intervals (Mauris et al., 2000). The latter approach is adapted in the context of the application where the customers' evaluations are expressed with stars translated into score-bounded intervals. In fact, our proposition is based on the fact that a possibility distribution $\pi$ (with $\Pi$ the associated possibility measure and $N$ the associated necessity measure) defines a family of probability distributions $P$ that it dominates i.e., $\forall A \subset \mathrm{R}, N(A) \leqslant P(A) \leqslant \Pi(A)$. Thus it can represent situations where $P$ is not precisely known, but only in an approximate way (Mauris et al., 2000).

From our possibilistic interpretation, $m_{i}^{l}$ is now considered as an uncertain value represented by a possibility distribution $\pi_{i}^{l}$. For the sake of simplicity and clarity for the user (manager, marketing team of EC, etc.), we choose to consider the case of piecewise linear distributions: to each criterion a trapezoidal or triangular possibility distribution $\pi_{i}$ is associated. $\pi_{i}$ is thus defined by four parameters $(a, b, c, d)$ with the interval $[b, c]$ as kernel and the interval $[a, d]$ as support. For a triangular distribution, the kernel is reduced to one point $(b=c)$. In the case of multi-modal distributions, the distribution is defined as unions of parameters, e.g. $(a, b, c, d)$ and $\left(a^{\prime}, b^{\prime}, c^{\prime}, d^{\prime}\right)$ for a bimodal distribution (Fig. 3).

The evaluations proposed by "ciao.com" vary from 0 to 5 stars (resp. rectangular bars) (Fig. 4). The sensitivity of the evaluations ranges between a quarter of a star and a star depending on the cases. As the satisfaction scales of the various users are not "calibrated" (and thus not completely commensurate), stars/bars are modelled by overlapped intervals to take this effect into account.

Each partial evaluation (star or bar) is then translated into a score bounded-interval as shown in Table 2. For example, to a score of $2.25 \mathrm{stars} / \mathrm{bars}$, we consider that we can indifferently associate a numerical value from 5 to 9 according to the customer' scale. This set of overlapped intervals is then approximated by a possibility distribution according to a method developed by Dubois and Prade (1988) and previously applied in Mauris et al. (2000).

For the sake of simplicity, we only consider three evaluation criteria in our illustration example: Product and service pricing (1); Range of services and products (2); and Purchase tracking function (3). From customers' evaluations histograms relative to each of these three criteria (Fig. 5a), the corresponding possibility distributions are represented in Fig. 5b. In this example, Product and service pricing is a bimodal distribution. Both others are mono modal. An explanation of the bimodality for Product and service pricing criterion might be: the EC has very interest- 


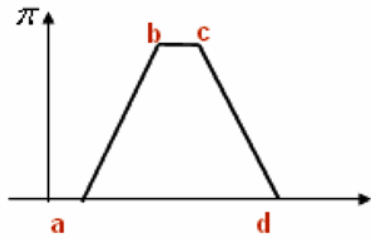

Multi modal representation
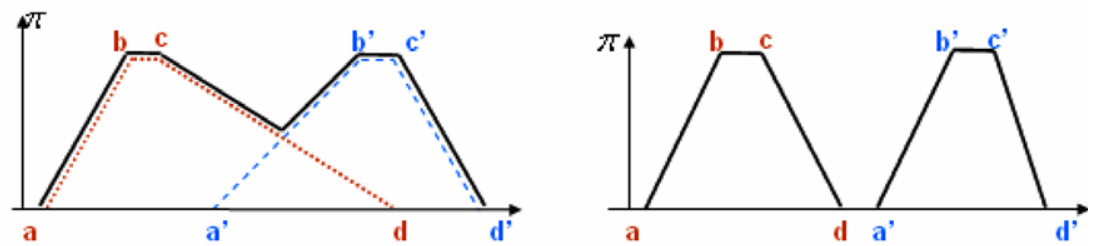

Fig. 3. Parameterized representation.

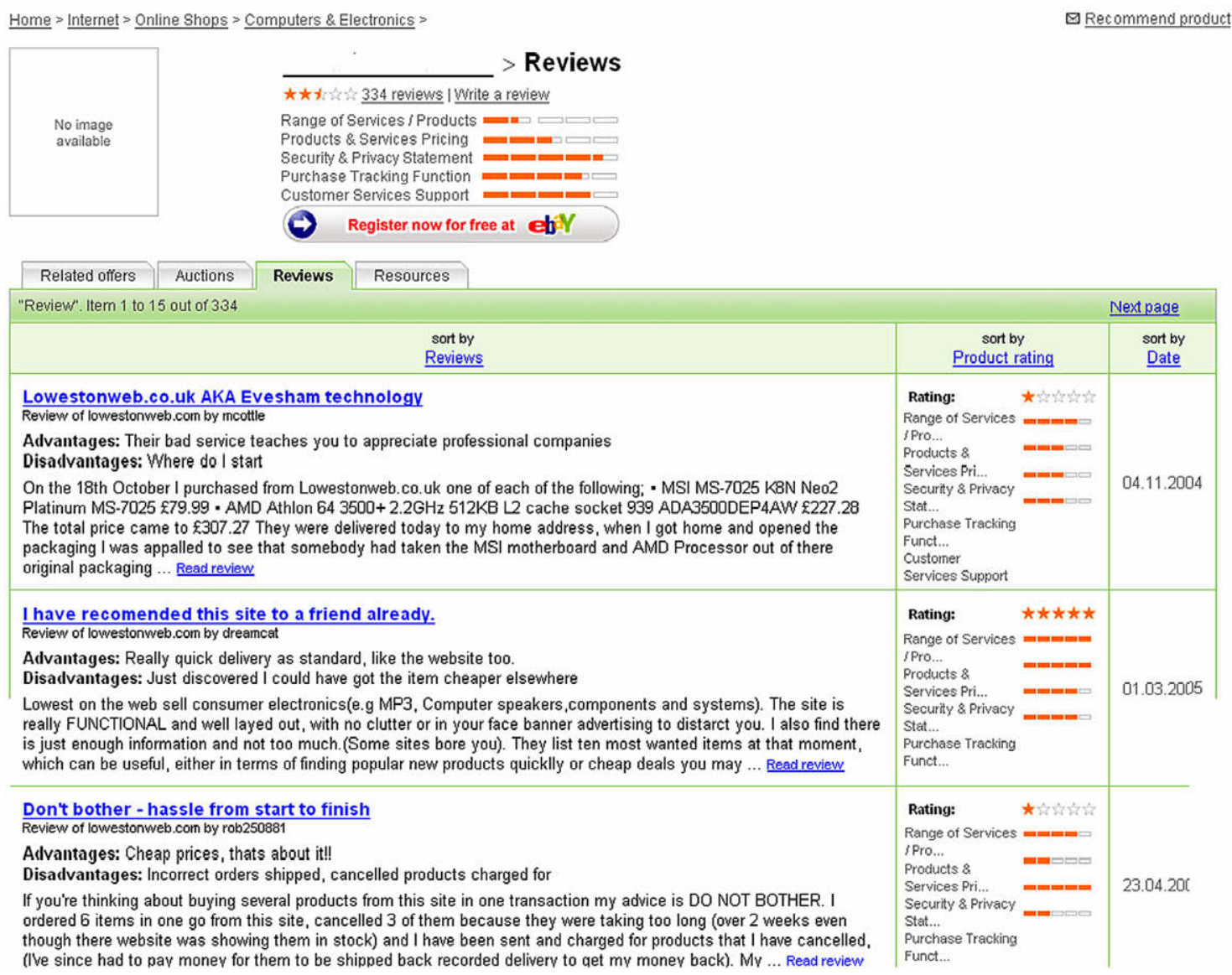

Fig. 4. "Tochibiya.com" evaluations in "ciao.com".

ing prices w.r.t. DVDs, CDs, etc., but it is much less competitive concerning high-tech products.

\subsection{Decision aiding indicators}

The representation in terms of possibility distribution may sometimes appear as a thorny mathematical artefact for decision makers. This mathematical meaning may cause some interpretation troubles for decision-makers. That's why decision-making indicators have been introduced to describe possibility distributions in a more interpretable and intuitive manner. Decision-makers can indifferently choose reasoning directly either on distributions or on their indicators for decision-making.

\subsubsection{Definitions}

Concise easily interpretable pieces of information, such as average, variability or controversial aspects of the 
Table 2

Translation of partial evaluations (star or bar) into bounded-intervals

\begin{tabular}{llll}
\hline Stars/bars & Interval & Stars/bars & Interval \\
\hline 0.25 & $0-1$ & 2.75 & $7-8-9-10-11$ \\
0.5 & $0-1-2$ & 3 & $8-9-10-11-12$ \\
0.75 & $0-1-2-3$ & 3.25 & $9-10-11-12-13$ \\
1 & $0-1-2-3-4$ & 3.5 & $10-11-12-13-14$ \\
1.25 & $1-2-3-4-5$ & 3.75 & $11-12-13-14-15$ \\
1.5 & $2-3-4-5-6$ & 4 & $12-13-14-15-16$ \\
1.75 & $3-4-5-6-7$ & 4.25 & $13-14-15-16-17$ \\
2 & $4-5-6-7-8$ & 4.5 & $4-15-16-17-18$ \\
2.25 & $5-6-7-8-9$ & 4.75 & $15-16-17-18-19$ \\
2.5 & $6-7-8-9-10$ & 5 & $16-17-18-19-20$ \\
\hline
\end{tabular}

customers' opinions, are useful notions to the EC marketing team. In this view, we propose to describe possibility distributions using indicators of location, imprecision and divergence.

Let us recall that a possibility distribution $\pi$ (with $\Pi$ the associated possibility measure and $N$ the associated necessity measure) defines a family of probability distributions
$P$ that it dominates, i.e. $\forall A \subset \mathrm{R}, N(A) \leqslant P(A) \leqslant \Pi(A)$. Two extreme probability distributions of this family are of particular interest, $P^{*}$ defined by its cumulative probability function $F^{*} / \forall x \in \mathbf{R}, F^{*}(x)=N(]-\infty, x[)$, and $P^{*}$ defined by its cumulative probability function $F^{*} / \forall x \in \mathbf{R}$, $\left.\left.F^{*}(x)=\Pi(]-\infty, x\right]\right)$.

Our proposition for the decision aiding indicators is based upon the definition of the upper $\left(E^{*}\right)$ and lower $\left(E^{*}\right)$ values of the mean value of these probability distributions (Dubois and Prade, 1987; Fortemps and Roubens, 1996):

$E^{*}(\pi)=\int_{-\infty}^{+\infty} x \mathrm{~d} F_{*}(x) \mathrm{d} x \quad$ and $\quad E_{*}(\pi)=\int_{-\infty}^{+\infty} x \mathrm{~d} F^{*}(x) \mathrm{d} x$.

In fact $\left[E^{*}(\pi), E^{*}(\pi)\right]$ defines an interval containing all the mean values computed according to all the probability distribution functions $P$ of the family. Let us still note that $E^{*}(\pi)$ and $E^{*}(\pi)$ are invariant by linear transformations (Dubois and Prade, 1987). This important property will be the key to the indicator definitions and to the criterion contribution expression.
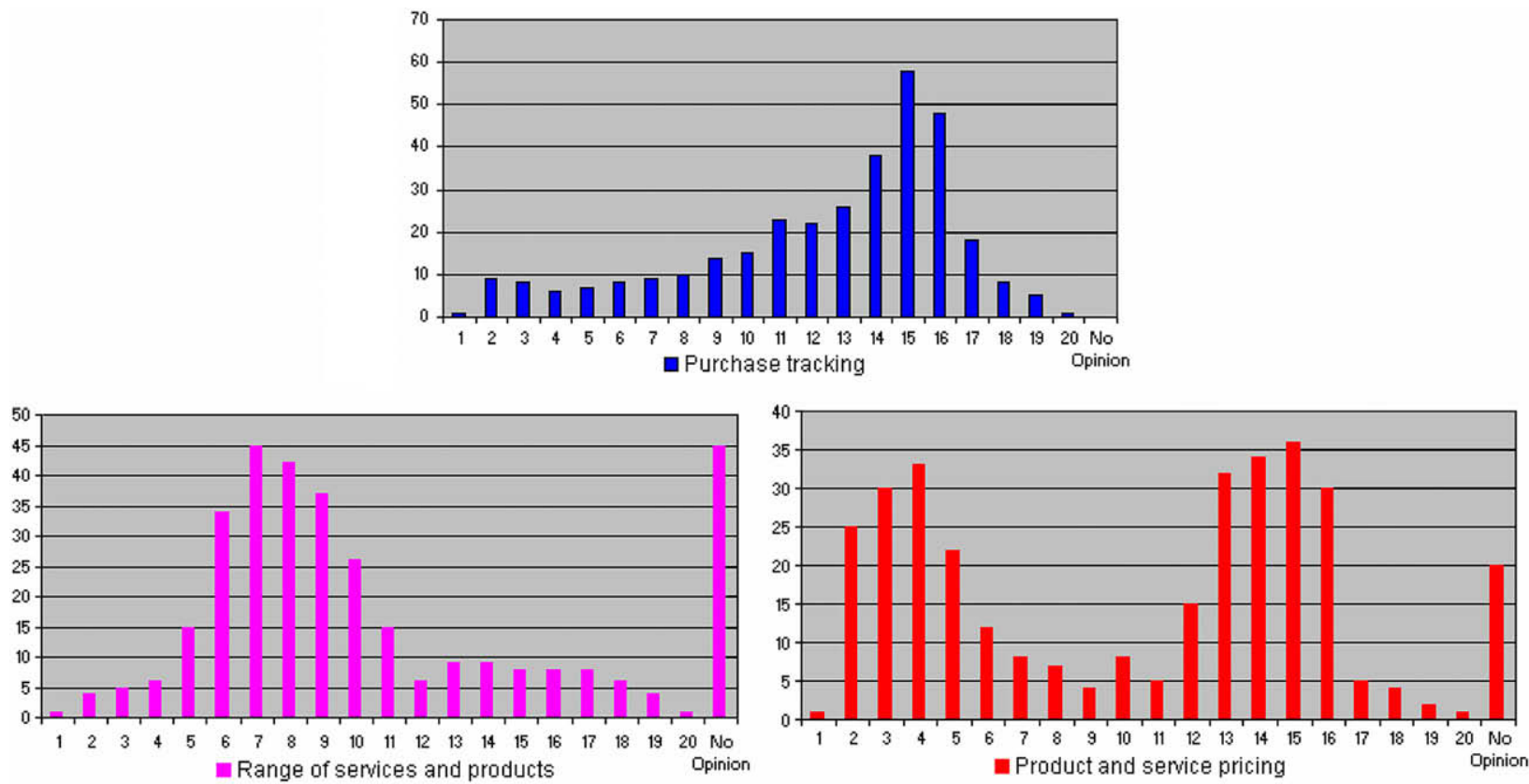

Fig. 5a. Histograms representing customers' evaluations for "Tochibiya.com" in ciao.com.

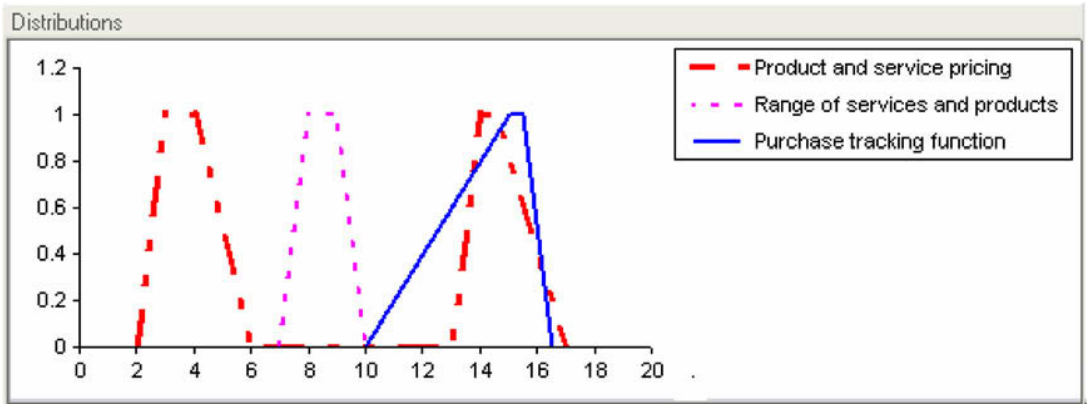

Fig. 5b. Criteria distributions of "Tochibiya.com". 
Table 3

Expressions of the marketing diagnosis indicators: location and imprecision indicators

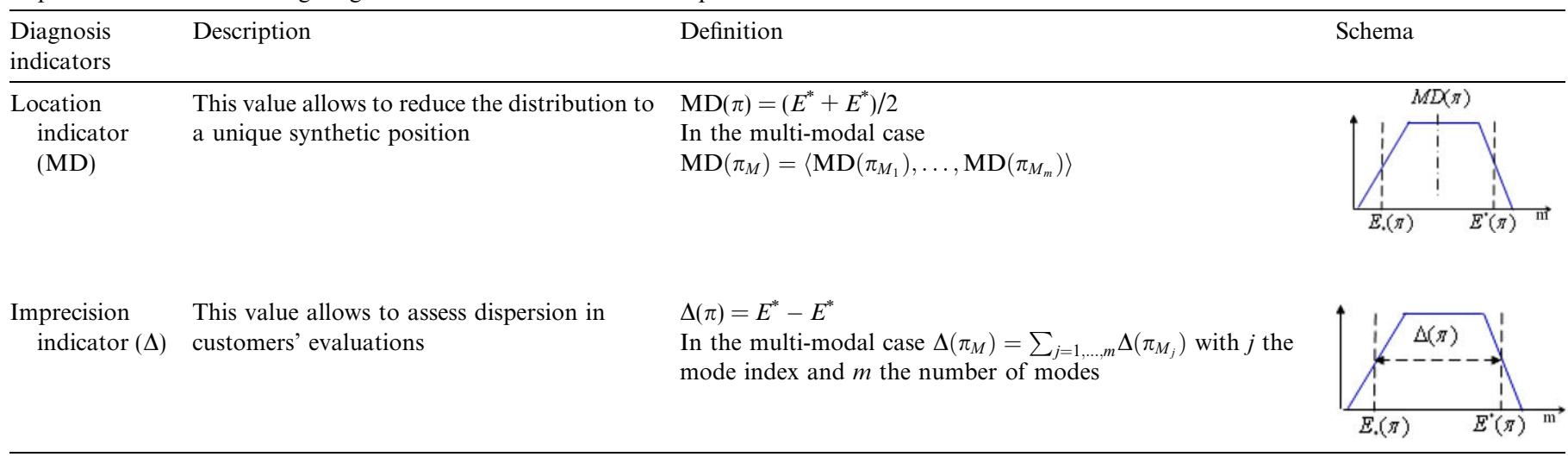

\subsubsection{Location, imprecision and divergence diagnosis indicators in marketing activities}

To simplify the manager's interpretation of distributions, we propose to describe each distribution by a location, an imprecision and a divergence indicator. In this paper, we focus on the divergence indicator because it appears as very relevant in customers' feedback analysis. Indeed, detecting a divergence of opinions in the evaluation of the EC and identifying the criteria that explain it, are crucial for marketing teams to better target their customers. A divergence indicator is also of much less common use than the two others - location and imprecision - that are equivalent to average and standard deviation items in probabilities, whereas multimodality is more rarely dealt with in the literature.

Though our main concern is the divergence indicator, the location and imprecision indicators are briefly presented in Table 3. More details are available in Denguir et al. $(2006 a, b)$.

The divergence indicator reflects a more or less important degree of controversy in customers' evaluations. It is translated by a multi-modal distribution $\pi_{M}$, with $m \geqslant 2$ the number of modes. $\pi_{M}$ is defined as the union of the mono modal distributions $\pi_{M}=\bigcup_{j=1, \ldots, m} \pi_{M^{j}}$. This multimodality can appear under two possible forms. In the first case, the distribution modes are partially disconnected (shape a-Fig. 6). In the second case, the modes are totally disconnected (shape b-Fig. 6). In the "shape a" case, an additional distribution is proposed: the multi-modal distri- bution $\pi_{M}$ ( $m=2$ in Fig. 6a) is disjointed into $m$ mono modal distributions $\left(\left(\pi_{M^{21}}\right)_{D}\right.$ and $\left.\left(\pi_{M^{22}}\right)_{D}\right)$ as shown in Fig. 6.

Furthermore, to each multi-modal distribution, an envelope distribution $\left(\pi_{\text {env }}\right)$ is associated (Fig. 7). Let $\Delta\left(\pi_{\text {env }}\right)$ be its imprecision indicator. We have the following relation:

$\Delta\left(\pi_{\text {env }}\right)=E^{*}\left(\pi_{\text {env }}\right)-E_{*}\left(\pi_{\text {env }}\right)=E^{*}\left(\pi_{M_{m}}\right)-E_{*}\left(\pi_{M_{1}}\right)$.

Definition. The divergence indicator Div of a distribution $\pi$ is defined as the difference between the average imprecision of its corresponding envelope distribution and its own average imprecision:

$\operatorname{Div}(\pi)=\Delta\left(\pi_{\text {env }}\right)-\Delta(\pi)$.

We have: $\forall \pi, \Delta\left(\pi_{\text {env }}\right) \geqslant \Delta(\pi)$, this implies that $\forall \pi$, $\operatorname{Div}(\pi) \geqslant 0$.

Let us consider again the example of Tochibiya.com in Fig. 5. Criterion (1), the Product and service pricing criterion is bimodal in this illustration. Range of services and products (2) and Purchase tracking function (3) are mono modal. Product and service pricing criterion is at the origin of a divergence in the customers' opinions. The corresponding envelope distribution is $(2,3,14.5,17)$ with an imprecision of $\Delta_{\text {lenv }}=13.25$ (Eq. (1)). Definitions in Table 3 and Eq. (2) provide the values of the indicators summarized in Table 4.

The Product and service pricing criterion is characterized by an important divergence (8.50). Although, "Toc-

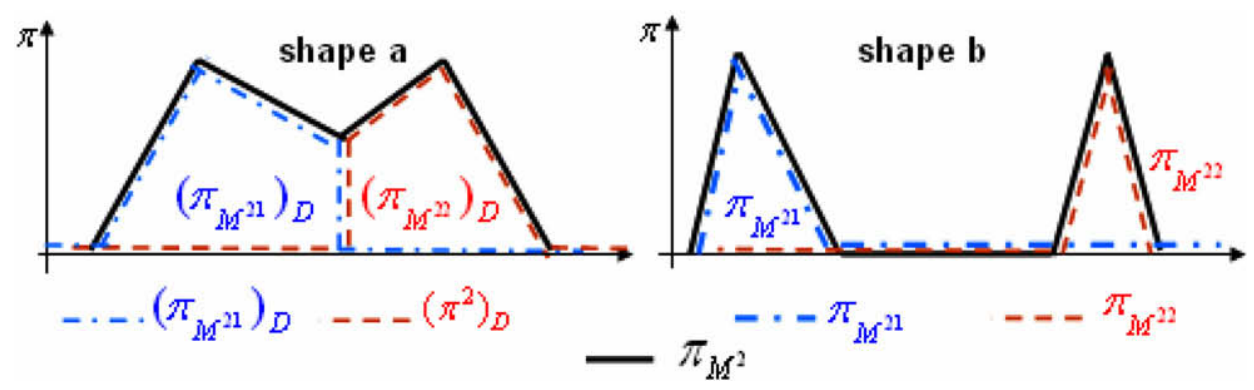

Fig. 6. Shapes of the multi-modal distribution. 


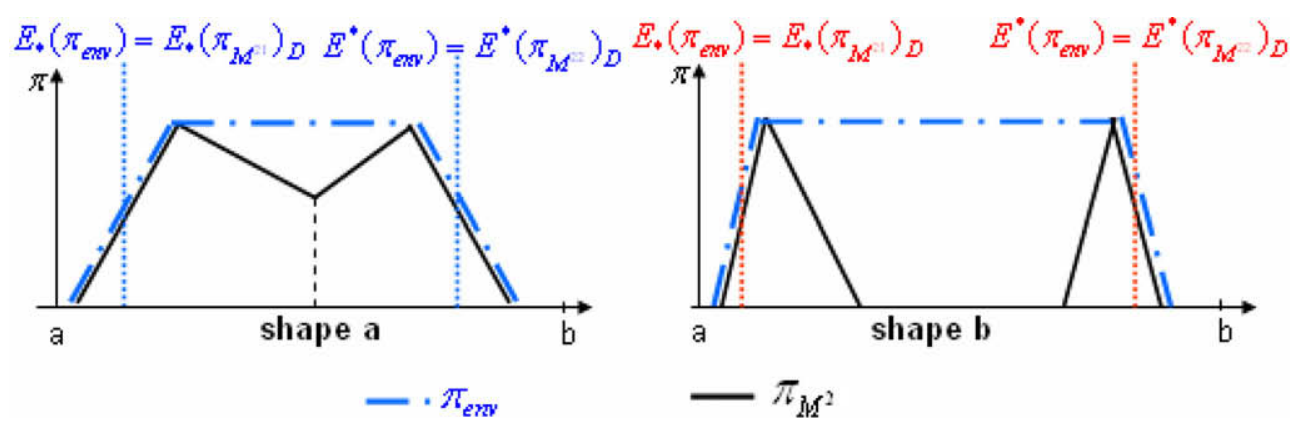

Fig. 7. Envelope distribution, case of bimodal distribution.

Table 4

Diagnosis indicators relative to Tochibiya.com criteria in ciao.com

\begin{tabular}{llll}
\hline & $\begin{array}{l}\text { Location indicator } \\
(\mathrm{MD})\end{array}$ & $\begin{array}{l}\text { Imprecision } \\
\text { indicator }(\Delta)\end{array}$ & $\begin{array}{l}\text { Divergence } \\
\text { indicator (Div) }\end{array}$ \\
\hline$(1)$ & $\langle 3.75 ; 14.625\rangle$ & 4.75 & 8.50 \\
$(2)$ & 8.50 & 2.00 & 0.00 \\
$(3)$ & 14.25 & 3.50 & 0.00 \\
\hline
\end{tabular}

hibiya.com" is a specialist in discounted products and its marketing strategy is based on decreasing prices, it remains evident that not all customers find the prices interesting. This characterization is richer than the misleading one proposed by "ciao.com" that would simply be "the score is between 8 and 9 (two stars and a half)" that reveals nothing about the way this mean value has been achieved, etc., we cannot know whether it results from a consensus, a dispersed set of opinions or, as it is the case in this example, from a singular controversy.

At this stage of the study, we have proposed a set of descriptive indicators according to a possibility distribution. Because diagnosis in marketing consists in identifying the criteria that explain a bad performance of the EC, we are now considering the propagation of indicators through the aggregation operator. This point is exposed in the following section.

\section{Multi-criteria aggregation of e-commerce evaluation in uncertain FBDS}

The aggregation issue consists in computing $\pi_{\mathrm{ag}}(m)$ for a given operator $H$ and $n$ partial distributions $\pi_{i}$. The propagation of the possibility distributions $\pi_{i}$ through the aggregation operator $H$ obeys Zadeh's extension principle (Zadeh, 1978).

$\pi_{\mathrm{ag}}(m)=\sup _{\left(m_{1}, \ldots, m_{n}\right) /_{H\left(m_{1}, \ldots, m_{n}\right)=m}}\left(\min \left(\pi_{1}\left(m_{1}\right), \ldots, \pi_{n}\left(m_{n}\right)\right)\right)$,

where $\pi_{\mathrm{ag}}(m)$ is the global evaluation of an EC and $\pi_{i}\left(m_{i}\right)$ its partial evaluation (possibility distribution) w.r.t. criterion $i$. In case of bimodal distribution propagation, the theorem of the union is used (Dubois and Prade, 1987).

In this paper, we are considering the most frequently used aggregation operator: the weighted arithmetic mean operator $\left(\mathrm{WAM}_{\omega}\right)$, with $\omega_{i}$ the relative importance of criterion $i$, such that $\sum_{i=1}^{n} \omega_{i}=1$.

When the partial scores to be aggregated are possibility distributions, the overall score of $\mathrm{EC}, s^{l}$, is written in the following linear expression:

$\operatorname{WAM}_{\omega}\left(\pi^{l}\right)=\pi_{\mathrm{ag}}^{l}=\sum_{i=1}^{n} \omega_{i} \pi_{i}^{l}$.

Identifying the aggregation strategy is an important issue. This strategy depends on the activity sector. For example, if we consider the case of an EC specialized in discount products, its objective is to sell more and more products, the criterion "price" is then important in the strategy modelling. A solution to support the marketing or benchmarking teams in the identification strategy may be to couple the FBDS with an identification tool like the one proposed in (Clivillé et al., 2007) that relies on the MACBETH method. As this point is not central in this paper, we will consider hereafter that this identification has led to the following importance coefficients: $\omega_{1}=0.5, \omega_{2}=0.3, \omega_{3}=0.2$. This means that assessing the cyber-consumers' satisfaction regarding the price is very important in the organisation strategy.

Fig. 8 represents the aggregated distribution of "Tochibiya.com" when the input partial distributions of the WAM are the ones proposed in Fig. 5.

We have shown in the last section that the display of the whole possibility distribution is of value in the diagnosis process of the customer satisfaction, but concise quantified

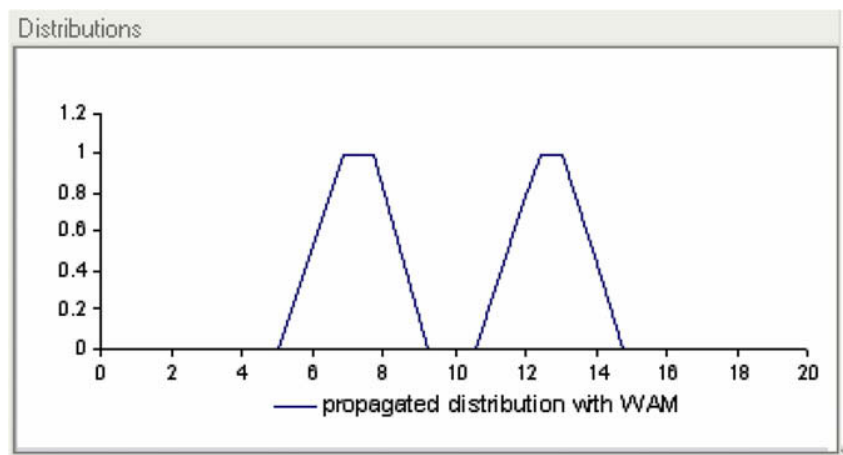

Fig. 8. The possibilistic global score of "Tochibiya.com". 
interpretable pieces of information reflecting variability or controversial aspects of the customers' opinions are also significant and useful diagnosis indicators. Thus, let us consider the propagation of these indicators through the WAM.

Let us first remark that we are interested in the divergence indicator only when the propagated distribution $\pi_{\mathrm{ag}}$ is multi modal ( $\pi_{\mathrm{ag} M}$, with $m$ modes $\left.m \geqslant 2\right)$. In fact we have:

$$
\begin{aligned}
\operatorname{Div}\left(\pi_{\mathrm{ag}}\right) \neq 0 & \Rightarrow \exists \pi_{i} / \operatorname{Div}\left(\pi_{i}\right)>0 \quad \text { but } \operatorname{Div}\left(\pi_{\mathrm{ag}}\right) \\
& =0 \nRightarrow \forall \pi_{i} / \operatorname{Div}\left(\pi_{i}\right)=0 .
\end{aligned}
$$

This means that even when the partial distributions are multi modal their propagation by an aggregation operator can lead to a mono modal aggregated distribution. It means that even when $\operatorname{Div}\left(\pi_{\mathrm{ag}}\right)=0$, we can have $\forall \pi_{i}$, $\operatorname{Div}\left(\pi_{i}\right) \neq 0$.

Explaining or diagnosing an indicator attached to $\pi_{\mathrm{ag}}$ consists in breaking down this indicator in terms of criteria contributions. Each indicator of $\pi_{\mathrm{ag}}$ can be rewritten as the sum of criteria contributions. The marginal contributions of a criterion to the overall evaluation of an EC are essential notions to marketing activities. Indeed, the marketing team of an e-commerce can thus estimate the impact or influence of each criterion to the activity of the EC in real time and determine the criteria on which the e-commerce activity should be improved in priority to improve its global evaluation. The contributions of a criterion $i$ relatively to location and imprecision indicators, denoted $C_{i \mathrm{MD}}$ and $C_{i \Delta}$ are given in Table 5.

We are now interested in the contribution of the criteria distributions to the divergence indicator of $\pi_{\mathrm{ag}}$ by the WAM operator. Firstly, let us consider the case when $\pi_{\mathrm{ag} M}$ is bimodal, i.e. composed of two parts $\pi_{\mathrm{ag} 1}$ and $\pi_{\mathrm{ag} 2}$.

Table 5

Criteria contribution relative to the diagnosis indicators (DI)

\begin{tabular}{lll}
\hline (DI) & DI of propagated distribution $\pi_{\mathrm{ag}}$ & Criteria contribution \\
\hline $\mathrm{MD}$ & $\mathrm{MD}\left(\pi_{\mathrm{ag}}\right)=\sum_{i=1}^{n} C_{i \mathrm{MD}}$ & $C_{i \mathrm{MD}}=\omega_{i \mathrm{MD}}\left(\pi_{i}\right)$ \\
$\Delta$ & $\Delta\left(\pi_{\mathrm{ag}}\right)=\sum_{i=1}^{n} C_{i \Delta}$ & $C_{i \Delta}=\omega_{i} \Delta\left(\pi_{i}\right)$ \\
\hline
\end{tabular}

Using Eqs. (1) and (2), we obtain the following expression for the divergence indicator of $\pi_{\mathrm{ag} M}$ (Fig. 9):

$\operatorname{Div}\left(\pi_{\mathrm{ag} M}\right)_{m=2}=E_{*}\left(\pi_{\mathrm{ag}^{2}}\right)-E^{*}\left(\pi_{\mathrm{ag}^{1}}\right)$.

Using

$\operatorname{MD}(\pi)=\left(E_{*}(\pi)+E^{*}(\pi)\right) / 2, \operatorname{Div}\left(\pi_{\mathrm{ag} M}\right)_{m=2}=2 \mathrm{MD}\left(\pi_{\mathrm{ag}^{2}}\right)-$ $2 \mathrm{MD}\left(\pi_{\mathrm{ag}}\right)-\Delta\left(\pi_{\mathrm{env}}\right)$.

Then, we have:

$$
\begin{aligned}
\operatorname{Div}\left(\pi_{\mathrm{ag} M}\right)_{m=2} & =\sum_{i=1}^{n} 2\left(C_{i \mathrm{MD}^{2}}-C_{i \mathrm{MD}^{1}}\right)-C_{i \Delta \mathrm{env}} \\
& =\sum_{i=1}^{n} C_{i \text { Div }},
\end{aligned}
$$

$C_{i \text { Div }}$ represents the contribution of criterion $i$ to the divergence of the propagated distribution $\pi_{\mathrm{ag}}$.

In the general case, when $\pi_{\mathrm{ag} M}$ is multi modal $(m \geqslant 2)$, we extend Eq. (6):

$$
\begin{aligned}
\operatorname{Div}\left(\pi_{\mathrm{ag} M}\right)= & 2 \operatorname{MD}\left(\pi_{\mathrm{ag}^{m}}\right)-2 \operatorname{MD}\left(\pi_{\mathrm{ag}^{1}}\right)-\Delta\left(\pi_{\mathrm{env}}\right) \\
& -\sum_{j=2}^{m-1} \Delta\left(\pi_{\mathrm{ag} i}\right)
\end{aligned}
$$

with $m \geqslant 2$ the number of modes of $\pi_{\mathrm{ag} M}$ and $j$ the mode index. Then:

$$
\begin{aligned}
\operatorname{Div}\left(\pi_{\mathrm{ag} M}\right) & =\sum_{i=1}^{n}\left[2\left(C_{i \mathrm{MD}^{m}}-C_{i \mathrm{MD}^{1}}\right)-C_{i \Delta \mathrm{env}}-\sum_{j=2}^{m-1} C_{i \Delta^{j}}\right] \\
& =\sum_{i=1}^{n} C_{i \text { Div }}
\end{aligned}
$$

$C_{i \mathrm{MD}}$ and $C_{i \Delta}$ are defined in Table 5.

Let us consider again our example of "Tochibiya.com" evaluated in ciao.com. Table 6 summarizes the indicators of the aggregated distribution attributed to "Tochibiya.com" with the selected WAM.

Table 6

Diagnosis indicators relative to the global score

\begin{tabular}{llll}
\hline Diagnosis indicators & MD & $\Delta$ & Div \\
\hline Global score distribution & $\langle 7.275 ; 12.7125\rangle$ & 4.98 & 2.95 \\
\hline
\end{tabular}
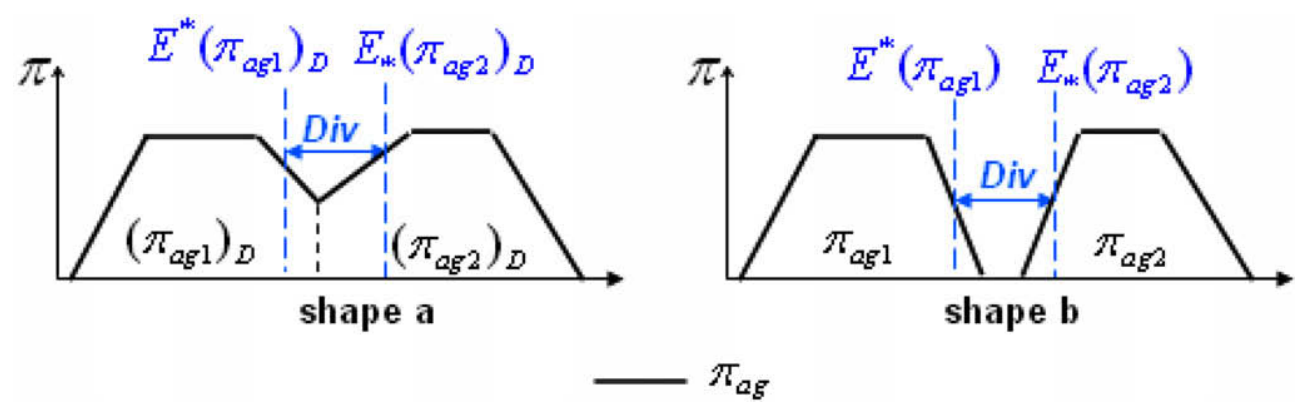

Fig. 9. Divergence indicator in bimodal case. 
Table 7

Criteria contribution to the divergence of the global score

\begin{tabular}{lrr}
\hline Criteria & $C_{i \mathrm{Div}}$ & $\begin{array}{l}\operatorname{Div}\left(\pi_{\mathrm{ag} M}\right)=\sum_{i=1}^{n} C_{i \mathrm{Div}} \\
4.15-0.63-0.57=2.95\end{array}$ \\
\hline Product and service pricing (1) & 4.15 & \\
Range of services and products (2) & -0.63 & \\
Purchase tracking function (3) & -0.57 & \\
\hline
\end{tabular}

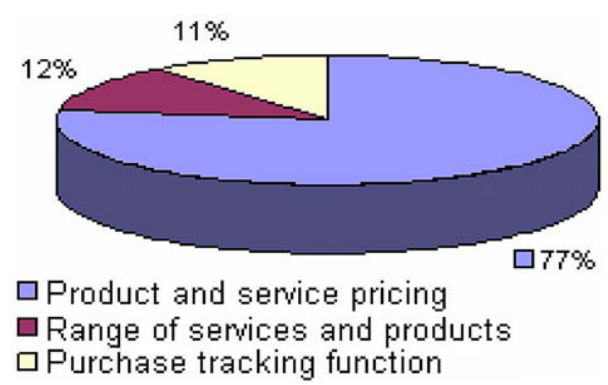

Fig. 10. Criteria contribution in percentage.

Now, the marketing team of "Tochibiya.com" would like to know the impact of the divergence on criterion (1) to the aggregated result. In Fig. 8, “Tochibiya.com" aggregated distribution presents a bimodality.

In Table 7, it can be checked that the divergence indicator of the global distribution of "Tochibiya.com" is smaller than the one of criterion 1 (2.95 instead of 8.5 (Table 4)). The marketing team needs an explanation of how this indicator has been smoothed. To explain this result let us assess the contribution of each criterion to the divergence of $\pi_{\mathrm{ag} M}$ (Eq. (6)) in Table 7.

The two mono modal distributions have smoothed the divergence of $\pi_{\mathrm{ag} M}$. In fact, the aggregation operator can only increase the imprecision, that will necessarily induce a smoothing of the input indicators: this is found in the sign of the contribution in a qualitative way and in a quantitative way we have $-0.63>-0.57$. This result is translated in terms of percentage in the Man-Machine Interface in Fig. 10.

It is clear that the distribution of the global score is much less divergent than the one of the Product and service pricing criterion. However, the marketing team has to propose some elements of solution to remove this divergence for competitive reasons. One recommendation that the marketing team can propose to the managers of the e-commerce organisation is to revise their pricing strategy in priority.

In further analysis, they must understand the reasons of such a divergence and try to get a more consensual appraisal for their EC. One way could be to contact some of the customers not satisfied with the product and service pricing and to understand the reasons of their dissatisfaction.

The diagnosis of the origin of the divergence relative to the Product and service pricing criterion has led to the identification of two classes of customers. The first one is a group of customers identified as specialists in computers' products; they have a large knowledge about existing products characteristics and pricing. This community may be composed in majority of 20-35-year-old persons. The second group is composed of non specialist and occasional customers. They have little or no information about computer products. This kind of information that represents a relevant and significant result for the marketing team in charge of diagnosing the EC activities, does not exist in ciao.com in its current form. The mere statistical evaluations they propose cannot support such diagnosis analysis.

\section{Conclusion}

This paper presents a system of multi-criteria evaluation of e-commerces, the Feedback Based Diagnosis System (FBDS). It is dedicated to marketing teams when trying to analyse the feedbacks and/or the evaluations of cyber consumers gathered in classical recommenders systems to diagnose the activity of their EC. The strategy of evaluation of the marketing team is reflected by an aggregation model which is a mere WAM. The FBDS also manages various aspects of uncertainty inherent to any collective process of evaluation by e-consumers. Thus, a WAM aggregation in a possibilistic description of the cyber-consumers' evaluations is proposed. Then, diagnosis indicators are provided to supply the marketing teams with some explanative elements to understand the degrees of satisfaction attributed to their site in the collective evaluation process. Quantitative analyses of the contributions of the decision-making indicators to the global evaluation of the EC are also proposed, with a specific focus on the divergence indicator related to the presence of different customers classes.

Further developments concern refining the aggregation model to reflect a more realistic model of customers' satisfaction. Thus, the next step is to extend all these results to an aggregation operator that enables to capture interactions between criteria in the aggregation model, such as the Choquet integral.

\section{References}

Berrah, L., Mauris, G., Vernadat, F., 2004. Information aggregation in industrial performance measurement: rationales, issues and definitions. International Journal of Production Research 42 (20), 4271-4293.

Berrah, L., Mauris, G., Montmain, J., 2008. Monitoring the improvement of an overall industrial performance based on a Choquet integral aggregation. The International Journal of Management Science OMEGA 36 (3), 340-351.

Breese, J.S., Heckerman, D., Kadie, C., 1998. Empirical analysis of predictive algorithms for collaborative filtering. In: 14th Conference on Uncertainty in Artificial Intelligence. Madison, USA, pp. 43-52.

Clivillé, V., Berrah, L., Mauris, G., 2007. Quantitative expression and aggregation of performance measurements based on the Macbeth multi-criteria method. International Journal of Production Economics 105 (1), 171-189.

Denguir, R.A., Mauris, G., Montmain, J., 2006a. Propagation of uncertainty by the possibility theory in a Choquet integral based decision making for an E-commerce website choice support. IEEE Transactions on Instrumentation and Measurement 55 (3), 721-728. 
Denguir, R.A., Montmain, J., Mauris, G., 2006b. E-commerce evaluations comparison and recommendation within a possibilistic framework. In: The Proceedings of the 11th IPMU International conference on Information Processing and Management of Uncertainty in Knowledge-Based Systems (IPMU'06). Paris, France.

Dubois, D., Prade, H., 1987. The mean value of a fuzzy number. Fuzzy Sets and Systems 24 (3), 279-300.

Dubois, D., Prade, H., 1988. Théorie des possibilités. Application à la représentation des connaissances en informatique.

Dubois, D., Foulloy, L., Mauris, G., Prade, H., 2004. Probability possibility transformations, triangular fuzzy sets and probabilistic inequalities. Reliable Computing 10 (4), 273-297.

Fortemps, P., Roubens, M., 1996. Ranking defuzzification methods based on area compensation. Fuzzy Sets and Systems 82 (3), 319-330.

Grigoroudis, E., Siskos, Y., 2002. Preference disaggregation for measuring and analyzing customer satisfaction: the MUSA method. European Journal of Operational Research 143, 148-170.

Herlocker, J.L., Konstan, J.A., Riedl, J., 2000. Explaining collaborative filtering recommendations. In: International Conference on Computer Supported Cooperative Work, Philadelphia, PA.

Kwan, I.S.Y., Fong, J., Wong, H.K., 2005. An e-customer behaviour model with online analytical mining for internet marketing planning. Decision Support Systems 41, 189-204.

Lang, K., 1995. NewsWeeder: Learning to filter netnews. In: Machine Learning 12th International Conference, Lake Tahoe, CA.

Leadershipfactor, 2004. The guide to customer satisfaction measurement.

Mauris, G., Berrah, L., Foulloy, L., Haurat, H., 2000. Fuzzy handling of measurement errors in instrumentation. IEEE Transactions on Instrumentation and Measurement 49, 89-93.
Mcnee, S., Lam, S., Guetzlaff, C., Konstan, J., Riedl, J., 2003. Confidence displays and training in recommender systems. In: International Conference on Human-Computer Interaction, pp. 176-183.

Mukherjee, R., Dutta, P.S, Sen S., 2001. Movies2go - a new approach to online movie recommendations. In: IJCAI workshop on "Intelligent Techniques for Web Personalisation".

Pazzani, M., Muramatsu, J., Billsus, D., 1996. Syskill \& Webert: Identifying interesting web sites. In: National Conference on Artificial Intelligence, Portland, Oregon.

Schafer, J., Konstan, J., Riedl, J., 1999. Recommender systems in ecommerce. In: Proceedings of the ACM'1999 Conference on Electronic Commerce.

Schafer, J., Konstan, J., Riedl, J., 2001. E-commerce recommendation applications. Journal of Data Mining and Knowledge Discovery.

Schafer, J., 2005. The Application of Data-Mining to Recommender Systems. Ph.D. Thesis, University of Northern Iowa.

Schniederjans, M.J., Cao, O., 2002. E-Commerce Operations Management. World Scientific.

Terveen, L., Hill, W., 1991. Beyond recommender systems: helping people help each other. In: Caroll, J. (Ed.), HCI in the Millennium. AddisonWesley, pp. 1-21.

Zadeh, L., 1978. Fuzzy sets as a basis for a theory of possibility. Fuzzy Sets and Systems 1, 3-28.

Zhaohao, S., Gavin, R., 2004. Intelligent Techniques in E-Commerce: A Case Based Reasoning Perspective (Studies in Fuzziness and Soft Computing). BERLIN Published.

Zollo, G., Iandoli, L., Cannavacciuolo, A., 1999. The performance requirements analysis with fuzzy logic. Fuzzy Economic Review 4, 35 69. 